\title{
On second order sufficient optimality conditions for a bang-singular arc
}

\author{
Laura Poggiolini ${ }^{* \dagger}$ \\ Università di Firenze \\ E-mail: laura.poggiolinieunifi.it
}

\section{Gianna Stefani ${ }^{\ddagger}$}

Università di Firenze

E-mail: gianna.stefanieunifi.it

\begin{abstract}
We consider a bang-singular normal extremal for a Mayer problem associated to a single-input control system affine in the control. We give sufficient conditions for the extremal to be a strong local minimizer. The conditions include the coercivity of the extended second variation associated to the problem restricted to the singular arc and regularity conditions on the bang arc. Remark that the non-negativity of the extended second variation is a necessary condition, when the normalized adjoint covector is unique, and its coercivity is sufficient to ensure that the singular arc is a local minimizer for the restricted problem. The paper is an attempt to give second order sufficient conditions for an extremal including a finite number of both bang and singular arcs.
\end{abstract}

Control Systems: Theory, Numerics and Applications

30 March - 1 April 2005

Rome

\footnotetext{
* Speaker.

${ }^{\dagger}$ A footnote may follow.

$¥$ A footnote may follow.
} 


\section{Introduction}

The paper describes a work in progress aiming to give suffi cient conditions for a bang-singular Pontryagin extremal to be a strong local optimizer for the Mayer Problem

$$
\operatorname{minimize} \alpha(\xi(0))+\beta\left(\xi\left(T_{f}\right)\right)
$$

subject to

$$
\begin{aligned}
& \dot{\xi}(t)=f_{0}(\xi(t))+u f_{1}(\xi(t)) \quad t \in\left[0, T_{f}\right] \\
& \xi(0) \in N_{0}, \quad \xi\left(T_{f}\right) \in N_{f}, \\
& u \in[-1,1] .
\end{aligned}
$$

The state space is a smooth $n$-dimensional manifold $M$, and $f_{0}, f_{1}: M \rightarrow T M$ are smooth vector fi elds, by smooth we mean $C^{\infty}$.

Suppose to have a Pontryagin normal extremal, i.e. a couple $(\widehat{\xi}, \widehat{u})$, with associated adjoint covector

$$
\widehat{\lambda}: t \in\left[0, T_{f}\right] \rightarrow \widehat{\lambda}(t) \in T^{*} M,
$$

satisfying Pontryagin Maximum Principle (PMP). Recall that

$$
\pi \widehat{\lambda}(t)=\widehat{\xi}(t), \quad \forall t \in\left[0, T_{f}\right]
$$

where $\pi: T^{*} M \rightarrow M$ is the canonical projection, and $\widehat{\lambda}$ is a solution of the Hamiltonian system associated to the reference Hamiltonian - with appropriate transversality conditions - and it satisfi es the maximization property. To be more precise, if we denote by $H_{i}, i=0,1$, the Hamiltonian function associated to the vector fi eld $f_{i}$, that is

$$
H_{i}: \ell \in T^{*} M \rightarrow\left\langle\ell, f_{i}(\pi \ell)\right\rangle, \quad i=0,1,
$$

then $\widehat{\lambda}$ satisfi es the Hamiltonian system associated to

$$
\widehat{H}_{t}=H_{0}+\widehat{u}(t) H_{1},
$$

and

$$
\begin{aligned}
& \widehat{\lambda}(0)=\mathrm{d} \alpha(\widehat{\xi}(0)) \text { on } T_{\widehat{\xi}(0)} N_{0}, \quad \widehat{\lambda}\left(T_{f}\right)=-\mathrm{d} \beta\left(\widehat{\xi}\left(T_{f}\right)\right) \text { on } T_{\widehat{\xi}\left(T_{f}\right)} N_{f}, \\
& \left.\left.\widehat{u}(t) H_{1} \widehat{\lambda}(t)\right)=\max _{u \in[-1,1]} u H_{1} \widehat{\lambda}(t)\right) \quad \text { a.e. } t \in\left[0, T_{f}\right] .
\end{aligned}
$$

Therefore if $\left.H_{1} \widehat{\lambda}(t)\right) \neq 0$, then

$$
\left.\widehat{u}(t)=\operatorname{sgn}\left(H_{1} \widehat{(\lambda}(t)\right)\right) .
$$

An arc of an extremal is called "bang", if the associated control is either 1 or -1 and it is called singular if $H_{1}$ is zero on the associated adjoint covector.

This paper is part of a general research program aiming to extend the use of Hamiltonian methods in the study of second order suffi cient conditions for $\widehat{\xi}$ to be a strong local minimizer for 
the problem. Here strong local optimizer means optimal with respect to a neighborhood of $\widehat{\xi}$ in $C\left(\left[0, T_{f}\right], M\right)$, independently on the value of the control.

If $\widehat{\xi}$ is bang-bang with a fi nite number of switching times, say $t_{1}, \ldots, t_{r}$, then it is possible to defi ne a second variation $J^{\prime}$ associated to the fi nite-dimensional problem obtained by moving the switching times. In this case PMP implies

$$
H_{1}\left(\widehat{\lambda}\left(t_{i}\right)\right)=0 \quad i=1, \ldots, r
$$

and, under the strict bang-bang Legendre condition

$$
\left\langle\widehat{\lambda}\left(t_{i}\right),\left[f_{0}, f_{1}\right]\left(\widehat{\xi}\left(t_{i}\right)\right)\right\rangle \neq 0 \quad i=1, \ldots, r
$$

$J^{\prime \prime}>0$ is a suffi cient condition for the strong local optimality of the trajectory, see [2] and the reference therein.

If $\widehat{\xi}$ is totally singular, then it is possible to defi ne an extended second variation, whose coercivity is again a suffi cient condition for the strong local optimality of the trajectory, see [6].

Here we study the case when $\widehat{\xi}$ is the concatenation of a bang and a singular arc, namely we suppose that there is $T_{b} \in\left(0, T_{f}\right)$ such that

$$
\begin{array}{ll}
\widehat{u}(t) \equiv 1 & \forall t \in\left(0, T_{b}\right), \\
\widehat{u}(t) \in(-1,1) & \forall t \in\left(T_{b}, T_{f}\right) .
\end{array}
$$

Nothing changes if the bang control is identically equal to -1 .

Generally speaking, the Hamiltonian approach to suffi cient conditions to strong optimality consists in using the coercivity of a suitable second variation to construct a fi eld of non-intersecting state extremals (super-extremals) covering a neighborhood of the given trajectory. Such a fi eld is obtained by projecting on the state manifold the flow associated to the maximized Hamiltonian $H^{\max }$ (of a Hamiltonian $H \geq H^{\max }$ ) emanating from a suitable horizontal Lagrangean sub-manifold. The sub-manifold can be obtained, if the second variation is coercive, adding a suitable penalty which allows to reduce the problem to another one without constraints on the initial point. Moreover the fi eld of extremals can be constructed via the coercivity of the second variation of the free-fi xed problem. In the case of variable fi nal point, the existence of a fi eld of non-intersecting extremals reduces the problem to a fi nite dimensional one and further conditions, coming from the coercivity of the second variation, give the result.

The described above Hamiltonian methods suggest to analyze, as a fi rst step, the case when the initial point is free, therefore in this paper we study the problem

$$
\operatorname{minimize} \alpha(\xi(0))+\beta\left(\xi\left(T_{f}\right)\right)
$$

subject to

$$
\begin{aligned}
& \dot{\xi}(t)=f_{0}(\xi(t))+u(t) f_{1}(\xi(t)) \quad t \in[0, T], \\
& \xi(0) \in M, \quad \xi\left(T_{f}\right) \in N_{f}, \\
& u(t) \in[-1,1] .
\end{aligned}
$$


A consequence of PMP is that

$$
\begin{array}{ll}
\left.H_{1}(\widehat{\lambda}(t))=\widehat{\lambda}(t), f_{1}(\xi(t))\right\rangle \equiv 0 & \forall t \in\left[T_{b}, T_{f}\right], \\
\left.H_{01} \widehat{\widehat{\lambda}}(t)\right)=\left\langle\widehat{\lambda}(t),\left[f_{0}, f_{1}\right](\widehat{\xi}(t))\right\rangle \equiv 0 & \forall t \in\left[T_{b}, T_{f}\right],
\end{array}
$$

where equation (1.4) is obtained from (1.3) differentiating with respect to $t$.

We state the following regularity assumptions

Assumption 1. The bang arc is regular in the interval $\left[0, T_{b}\right)$, i.e.

$$
\left.H_{1}(\widehat{\lambda}(t))=\widehat{\lambda}(t), f_{1}(\widehat{\xi}(t))\right\rangle>0 \quad \forall t \in\left[0, T_{b}\right) .
$$

Differentiating with respect to time at $t=T_{b}$ and using (1.3) and (1.4), we obtain

$$
\left.\widehat{\lambda}\left(T_{b}\right),\left(\left[f_{0},\left[f_{0}, f_{1}\right]\right]+\left[f_{1},\left[f_{0}, f_{1}\right]\right]\right)\left(\widehat{\xi}\left(T_{b}\right)\right)\right\rangle \geq 0,
$$

and we strengthen the condition requiring

\section{Assumption 2.}

$$
\left.\widehat{\lambda}\left(T_{b}\right),\left(\left[f_{0},\left[f_{0}, f_{1}\right]\right]+\left[f_{0},\left[f_{0}, f_{1}\right]\right]\right)\left(\widehat{\xi}\left(T_{b}\right)\right)\right\rangle>0 .
$$

The optimality of the reference trajectory implies that the singular arc is optimal for the following problem

$$
\operatorname{minimize} \alpha \circ \exp \left(-T_{b} f^{+}\right)\left(\xi\left(T_{b}\right)\right)+\beta\left(\xi\left(T_{f}\right)\right)
$$

subject to

$$
\begin{aligned}
& \dot{\xi}(t)=f_{0}(\xi(t))+u(t) f_{1}(\xi(t)) \quad t \in\left[T_{b}, T_{f}\right] \\
& \xi\left(T_{b}\right) \in M, \quad \xi\left(T_{f}\right) \in N_{f}, \\
& u(t) \in[-1,1] .
\end{aligned}
$$

Remark that the extended second variation of this problem, defi ned in [6], has to be non-negative if the adjoint covector is unique up to a positive constant. Therefore a natural assumption is to require that it is coercive. This last condition ensures that the singular arc is optimal for the problem (1.6) - 1.7).

Allowing the trajectory to vary only on $\left[0, T_{b}\right]$, we obtain that the bang arc is optimal for the free-fi xed sub-problem

$$
\operatorname{minimize} \alpha(\xi(0))
$$

subject to

$$
\begin{aligned}
& \dot{\xi}(t)=f_{0}(\xi(t))+u(t) f_{1}(\xi(t)) \quad t \in\left[0, T_{b}\right] \\
& \xi(0) \in M, \quad \xi\left(T_{b}\right)=\widehat{x}_{b}, \\
& u(t) \in[-1,1] .
\end{aligned}
$$

It is easily seen that this implies

$$
L_{f_{1}} L_{f_{1}} \alpha_{s}\left(\widehat{x}_{b}\right) \geq 0
$$


Remark that the strict version of the above inequality is a consequence of the coercivity of the extended second variation of problem (1.6) - (1.7), see Condition 1 in Section B. Moreover Assumptions 1 - 1 - 2 and $L_{f_{1}} L_{f_{1}} \alpha_{s}\left(\widehat{x}_{b}\right)>0$ are suffi cient conditions for the local optimality of the bang arc with respect to problem (1.8) - (1.9), see Proposition 2 .

Our suffi cient conditions for problem 1.]) - 1.7) include an extra regularity assumption:

Assumption 3. There is a neighborhood $V$ of $\widehat{\xi}\left(T_{b}\right)$ in $M$ such that, if $y \in V$ and $L_{f_{1}}\left(\alpha \circ \exp -T_{b} f^{+}\right)(y)=0$, then

$$
L_{\left[f_{0}, f_{1}\right]}\left(\alpha \circ \exp -T_{b} f^{+}\right)(y) \leq 0 .
$$

The main result of the paper is the following:

Theorem 1. Assume $(\widehat{\xi}(\cdot), \widehat{u}(\cdot))$ is a bang-singular Pontryagin extremal of the optimal control problem (1.1) - (1.2). Let Assumptions 1 , 目 and 3 be satisfied. If the extended second variation of the restricted problem (1.6) - (1.7), defined in (3.3) - (3.4), is coercive along the singular arc, then $(\widehat{\xi}(\cdot), \widehat{u}(\cdot))$ is a strict strong local minimizer.

\section{Notation and preliminary remarks}

We denote the reference vector fi eld by $\widehat{f}$, i.e.

$$
\widehat{f_{t}}= \begin{cases}f^{+} \equiv f_{0}+f_{1} & \text { if } t \in\left[0, T_{b}\right] \\ f_{0}+\widehat{u}(t) f_{1} & \text { if } t \in\left[T_{b}, T_{f}\right]\end{cases}
$$

and its flow from time 0 by

$$
\widehat{S}:(x, t) \mapsto \widehat{S}_{t}(x) .
$$

We denote the iterated Lie brackets of the vector fi elds $f_{0}$ and $f_{1}$ by

$$
f_{i_{1} i_{2} \ldots i_{k}} \equiv\left[f_{i_{1}},\left[\ldots\left[f_{i_{k-1}}, f_{i_{k}}\right] \ldots\right] \quad i_{j} \in\{0,1\}, \quad j=1, \ldots, k,\right.
$$

and the associated Hamiltonian functions by

$$
H_{i_{1} i_{2} \ldots i_{k}}: \ell \mapsto\left\langle\ell, f_{i_{1} i_{2} \ldots i_{k}}(\pi \ell)\right\rangle, \quad j=1, \ldots, k .
$$

The reference time-dependent Hamiltonian function is

$$
\widehat{H}_{t}=\left\{\begin{array}{ll}
H_{0}+H_{1} & t \in\left[0, T_{b}\right] \\
H_{0}+\widehat{u}(t) H_{1} & t \in\left[T_{b}, T_{f}\right]
\end{array},\right.
$$

while the maximized Hamiltonian

$$
H: \ell \mapsto \max \left\{H_{0}(\ell)+u H_{1}(\ell), u \in[-1,1]\right\}
$$

is given by

$$
H: \ell \mapsto \begin{cases}\left(H_{0}+H_{1}\right)(\ell) & H_{1}(\ell)>0 \\ H_{0}(\ell)=\widehat{H}_{t}(\ell) & H_{1}(\ell)=0 \\ \left(H_{0}-H_{1}\right)(\ell) & H_{1}(\ell)<0\end{cases}
$$


For each, possibly time-dependent, Hamiltonian function $H_{t}$, we denote the associated Hamiltonian vector fi eld by

$$
\vec{H}_{t}: T^{*} M \rightarrow T T^{*} M
$$

With this notation $\widehat{\lambda}:\left[0, T_{f}\right] \rightarrow T^{*} M$ is solution of

$$
\dot{\lambda}(t)=\overrightarrow{\widehat{H}}_{t}(\lambda(t)) \text { on }\left[0, T_{f}\right]
$$

and the transversality conditions are

$$
\widehat{\lambda}(0)=\mathrm{d} \alpha\left(\widehat{x}_{0}\right), \quad \widehat{\lambda}\left(T_{f}\right)=-\mathrm{d} \beta\left(\widehat{x}_{f}\right) \text { on } T_{\widehat{x}_{f}} N_{f},
$$

where $\widehat{x}_{0} \equiv \widehat{\xi}(0)$ and $\widehat{x}_{f} \equiv \widehat{\xi}\left(T_{f}\right)$. Without loss of generality we may assume

$$
\widehat{\lambda}\left(T_{f}\right)=-\mathrm{d} \beta\left(\widehat{x}_{f}\right) \text { on } T_{\widehat{x}_{f}} M \text {. }
$$

Let us also defi ne

$$
\widehat{\ell}_{0} \equiv \widehat{\lambda}(0), \widehat{\ell_{b}} \equiv \widehat{\lambda}\left(T_{b}\right), \widehat{\ell}_{f} \equiv \widehat{\lambda}\left(T_{f}\right), \widehat{x}_{b} \equiv \widehat{\xi}\left(T_{b}\right)=\pi \widehat{\ell}_{b}
$$

Since the assumptions of Theorem 11 imply the strict generalized Legendre condition (SGLC):

$$
H_{101}(\widehat{\lambda}(t))>0, \forall t \in\left[T_{b}, T_{f}\right],
$$

see Remark 1 in Section 3 , then we can describe the following geometric picture near the adjoint covector. Defi ne

$$
\begin{aligned}
& \Sigma=\left\{\ell \in T^{*} M: H_{1}(\ell)=0\right\}, \\
& S=\left\{\ell \in T^{*} M: H_{1}(\ell)=H_{01}(\ell)=0, H_{101}(\ell)>0\right\},
\end{aligned}
$$

then $\widehat{\lambda}\left(\left[T_{b}, T_{f}\right]\right) \subset S \subset \Sigma, \vec{H}_{1}$ is tangent to $\Sigma$ and transversal to $S$ and $\vec{H}_{01}$ is transversal to $\Sigma$. Moreover, differentiating (1.4), we get

$$
H_{001}(\widehat{\lambda}(t))+\widehat{u}(t) H_{101}(\widehat{\lambda}(t))=0, \forall t \in\left[T_{b}, T_{f}\right],
$$

hence

$$
\left.\widehat{u}(t)=\frac{-H_{001}}{H_{101}} \widehat{\lambda}(t)\right), \forall t \in\left[T_{b}, T_{f}\right] .
$$

\section{The singular arc}

We now consider sub-problem (1.6) - (1.7), namely we consider only the admissible couples $(\xi, u)$ such that $u(t)=\widehat{u}(t)=1$ for any $t \in\left[0, T_{b}\right]$. For this problem we use the results of [6].

Shifting time, denote

$$
\begin{aligned}
& T_{s}=T_{f}-T_{b}, \\
& \widehat{u}_{s}: t \in\left[0, T_{s}\right] \rightarrow \widehat{u}\left(t+T_{s}\right), \\
& \widehat{\xi}_{s}: t \in\left[0, T_{s}\right] \rightarrow \widehat{\xi}\left(t+T_{s}\right), \\
& \alpha_{s}:=\exp -T_{b} f^{+}, \\
& \widehat{\lambda}_{s}: t \in\left[0, T_{s}\right] \rightarrow \widehat{\lambda}\left(t+T_{s}\right) \\
& \widehat{\beta}_{s}:=\beta \circ \widehat{S}_{T_{f}} \circ \widehat{S}_{T_{b}}^{-1}, \\
& \gamma_{s}:=\alpha_{s}+\widehat{\beta}_{s} .
\end{aligned}
$$


By the properties of the adjoint covector, using (2.2), we get $\mathrm{d} \gamma_{s}\left(\widehat{x}_{b}\right)=0$, so that

$$
\gamma_{s}^{\prime \prime} \equiv \mathrm{d}^{2} \gamma_{s}\left(\widehat{x}_{b}\right)
$$

is a well defi ned quadratic form on $T_{x_{b}} M$.

With the above stated notations, the sub-problem (1.6) - (1.7) is equivalent to

$$
\operatorname{minimize} \alpha_{s}(\xi(0))+\beta\left(\xi\left(T_{s}\right)\right)
$$

subject to

$$
\begin{aligned}
& \dot{\xi}(t)=f_{0}(\xi(t))+u(t) f_{1}(\xi(t)) \quad t \in\left[0, T_{S}\right] \\
& \xi(0) \text { free, } \quad \xi\left(T_{S}\right) \in N_{f}, \\
& u(t) \in[-1,1]
\end{aligned}
$$

with reference couple $\left(\widehat{\xi}_{s}, \widehat{u}_{s}\right)$ and adjoint covector $\widehat{\lambda}_{s}$. Moreover letting

$$
\begin{aligned}
& \widehat{N}_{f}=\widehat{S}_{T_{b}} \circ \widehat{S}_{T_{f}}^{-1}\left(N_{f}\right), \\
& h_{t}(x)=\left(\exp T_{b} f^{+}\right)_{*}^{-1}\left(\widehat{S}_{t+T_{b}}\right)_{*}^{-1} f_{1}\left(\widehat{S}_{t+T_{b}} \circ \exp \left(-T_{b} f^{+}\right)(x)\right) \quad t \in\left[0, T_{s}\right],
\end{aligned}
$$

the second variation of problem [3.1), (3.2), as defi ned in [1], is given by the quadratic form on $T_{\widehat{x}_{b}} M \times L^{2}\left(\left[0, T_{s}\right]\right)$ defi ned below.

Proposition 1. For any $\delta e=(\delta x, v) \in T_{\widehat{x}_{b}} M \times L^{2}\left(\left[0, T_{s}\right]\right)$ the second order approximation of the cost is

$$
J_{s}^{\prime \prime}[\delta e]^{2}=\frac{1}{2} \gamma_{s}^{\prime \prime}[\delta x]^{2}+\int_{0}^{T_{s}} v(t) L_{\eta(t)} L_{h_{t}} \widehat{\beta}_{s}\left(\widehat{x}_{b}\right) \mathrm{d} t
$$

where $\eta$ satisfies

$$
\begin{aligned}
& \dot{\eta}(t)=v(t) h_{t}\left(\widehat{x}_{b}\right), \\
& \eta(0)=\delta x, \quad \eta(T) \in T_{\widehat{x}_{b}} \widehat{N}_{f} .
\end{aligned}
$$

Remark that $J_{s}^{\prime \prime}$ does not depend on the choice of $\beta$ with the property (2.2).

After the transformation

$$
\Phi: v \in L^{2}\left(\left[0, T_{s}\right]\right) \rightarrow\left(w_{0}, w\right)=\left(\int_{0}^{T_{s}} v(s) \mathrm{d} s, t \rightarrow \int_{t}^{T_{s}} v(s) \mathrm{d} s\right) \in \mathbb{R} \times L^{2}\left(\left[0, T_{s}\right]\right)
$$

we obtain an equivalent formulation for the second variation. Since $\Phi$ is dense and continuous, we consider its extension to all the space $T_{\widehat{x}_{b}} M \times \mathbb{R} \times L^{2}\left(\left[0, T_{s}\right]\right.$, which we call extended second variation $J_{e}^{\prime \prime}$, and which is given by

$$
\begin{aligned}
J_{e}^{\prime \prime}\left[\delta e_{s e}\right]^{2} & =\frac{1}{2} \gamma_{s}^{\prime \prime}\left(\widehat{x}_{b}\right)[\delta x]^{2}+\frac{w_{0}^{2}}{2} L_{f_{1}} L_{f_{1}} \widehat{\beta}_{s}\left(\widehat{x}_{b}\right)+w_{0} L_{\delta x} L_{f_{1}} \widehat{\beta}_{s}\left(\widehat{x}_{b}\right) \\
& +\frac{1}{2} \int_{0}^{T_{s}} w^{2}(t) H_{101}\left(\widehat{\lambda}_{s}(t)\right) \mathrm{d} t+\int_{0}^{T_{s}} L_{\zeta(t)} L_{w(t) h_{t}} \widehat{\beta}_{s}\left(\widehat{x}_{s}\right) \mathrm{d} t
\end{aligned}
$$


where $\varsigma$ satisfi es

$$
\begin{aligned}
& \varsigma(0)=\delta x+w_{0} f_{1}\left(\widehat{x}_{b}\right) \\
& \dot{\zeta}(t)=w(t) \dot{h}_{t}\left(\widehat{x}_{b}\right) \quad t \in\left[0, T_{s}\right] \\
& \varsigma\left(T_{s}\right) \in T_{\widehat{x}_{b}} \widehat{N}_{f} .
\end{aligned}
$$

Remark that

$$
\dot{h}_{t}=\left(\exp T_{b} f^{+}\right)_{*}^{-1}\left(\widehat{S}_{t+T_{b}}\right)_{*}^{-1}\left[f_{0}, f_{1}\right] \circ \widehat{S}_{t+T_{b}} \circ \exp \left(-T_{b} f^{+}\right) \quad t \in\left[0, T_{S}\right] .
$$

It is not diffi cult to prove, see also [§], that the Assumption " $J_{e}^{\prime}$ is coercive" is equivalent to the following two conditions:

Condition 1. $L_{f_{1}} L_{f_{1}} \alpha_{s}\left(\widehat{x}_{b}\right)>0$.

Condition 2. The coercivity of the quadratic form

$$
\begin{aligned}
J^{\prime \prime}\left[\delta y, w_{0}, w\right] & =\frac{1}{2} \gamma_{s}^{\prime \prime}\left(\widehat{x}_{b}\right)[\delta y]^{2}+\frac{w_{0}^{2}}{2} L_{f_{1}} L_{f_{1}} \widehat{\beta}_{s}\left(\widehat{x}_{b}\right)+w_{0} L_{\delta y} L_{f_{1}} \widehat{\beta}_{s}\left(\widehat{x}_{b}\right) \\
& +\frac{1}{2} \int_{0}^{T_{s}} w^{2}(t) H_{101}\left(\widehat{\lambda}_{s}(t)\right) \mathrm{d} t+\int_{0}^{T_{s}} L_{\zeta(t)} L_{w(t) \dot{h}_{t}} \widehat{\beta}_{s}\left(\widehat{x}_{b}\right) \mathrm{d} t
\end{aligned}
$$

where $\delta y$ and $\varsigma$ satisfy

$$
\begin{aligned}
& L_{\delta y} L_{f_{1}} \alpha_{s}\left(\widehat{x}_{b}\right)=0 \\
& \varsigma(0)=\delta y+w_{0} f_{1}\left(\widehat{x}_{b}\right), \\
& \dot{\zeta}(t)=w(t) \dot{h}_{t}\left(\widehat{x}_{b}\right) \quad t \in\left[0, T_{s}\right] \\
& \varsigma\left(T_{s}\right) \in T_{\widehat{x}_{b}} \widehat{N}_{f} .
\end{aligned}
$$

Remark 1. Condition 1 implies that $J^{\prime \prime}$ is an accessory linear-quadratic problem on $T_{\widehat{x_{b}}}$ M. Moreover the coercivity of $J^{\prime \prime}$ implies $S G L G$

$$
H_{101}\left(\widehat{\lambda}_{s}(t)\right)>0 \quad \forall t \in\left[0, T_{s}\right] .
$$

Remark 2. Since the initial point of our problem is free, then the normalized adjoint covector is unique, hence the conditions

$$
L_{f_{1}} L_{f_{1}} \alpha_{s}\left(\widehat{x}_{b}\right) \geq 0 \quad \text { and } \quad J^{\prime \prime} \geq 0
$$

are necessary for the optimality of $\widehat{\xi}_{\text {s }}$ in problem (3.1) - (3.2), and hence in the original problem.

If we defi ne

$$
M_{s} \equiv\left\{x \in M: L_{f_{1}} \alpha_{s}(x)=0\right\}
$$

it is clear that $\widehat{x}_{b} \in M_{s}$ and, by Condition 1 , that $f_{1}$ is transversal to $M_{s}$ in $\widehat{x}_{b}$. Therefore there exist a neighborhood $O\left(\widehat{x}_{b}\right)$ of $\widehat{x}_{b}$ in $M$, a positive number $\bar{b}$ and a neighborhood $O_{s}\left(\widehat{x}_{b}\right)$ of $\widehat{x}_{b}$ in $M_{s}$ such that the functions

$$
b: O\left(\widehat{x}_{b}\right) \rightarrow(-\bar{b}, \bar{b}) \quad \text { and } \quad y: O\left(\widehat{x}_{b}\right) \rightarrow O_{s}\left(\widehat{x}_{b}\right)
$$


are well defi ned by

$$
x=\exp b(x) f_{1}(y(x)) \quad \forall x \in O\left(\widehat{x}_{b}\right) .
$$

In [6] it is proved that defi ning $\widetilde{\alpha}_{s}: O\left(\widehat{x}_{b}\right) \rightarrow \mathbb{R}$ by

$$
\widetilde{\alpha}_{s}(x)=\alpha_{s}(y(x)),
$$

then $J^{\prime \prime}$ turns out to be the standard second variation of the following problem

$$
\operatorname{minimize} \widetilde{\alpha}_{s}(\xi(0))+\beta\left(\xi\left(T_{s}\right)\right)
$$

subject to

$$
\begin{aligned}
& \dot{\xi}(t)=\widehat{f}_{T_{b}+t}(\xi(t))+w(t)\left[f_{0}, f_{1}\right](\xi(t))+\frac{w^{2}(t)}{2}\left[f_{1},\left[f_{0}, f_{1}\right]\right](\xi(t)) \quad t \in\left[0, T_{s}\right] \\
& \xi(0) \text { free, } \quad \xi\left(T_{s}\right) \in N_{f}, \\
& u(t) \in[-1,1],
\end{aligned}
$$

with reference couple $(w, \xi)=\left(0, \widehat{\xi}_{s}\right)$.

From the results in [6], the coercivity of $J^{\prime \prime}$ implies, for any solution of system (3.2) belonging to a suitable neighborhood of the reference trajectory,

$$
\widetilde{\alpha}_{s}(\xi(0))+\beta\left(\xi\left(T_{s}\right)\right) \geq \widetilde{\alpha}_{s}\left(\widehat{x}_{b}\right)+\beta\left(\widehat{x}_{f}\right)=\alpha_{s}\left(\widehat{x}_{b}\right)+\beta\left(\widehat{x}_{f}\right),
$$

moreover equality holds if and only if $\xi=\widehat{\xi}_{s}$.

Remark 3. Since $J^{\prime \prime}$ is associated to a linear quadratic non singular control problem, then its coercivity can be checked via the non existence of conjugate times or through an appropriate Riccati equation, see for example [母], [П], [5] and the references therein.

Rewriting the result with respect to Problem (1.1) - (1.2), possibly restricting the neighborhood $O\left(\widehat{x}_{b}\right)$ we can state the following

Lemma 1. Let the extended second variation defined in (3.5) - (3.6) be coercive. There is a neighborhood $\mathcal{V}$ of the graph of $\widehat{\xi}_{\mid\left[T_{b}, T_{f}\right]}$ such that $\mathcal{V} \cap\left\{T_{b}\right\} \times M=O\left(\widehat{x}_{0}\right)$ and for any solution $\xi$ of (1.2) such that the graph of $\xi_{\left[T_{b}, T_{f}\right]}$ is contained in $\mathcal{V}$ the following inequality holds

$$
\widetilde{\alpha}_{s}\left(\xi\left(T_{b}\right)\right)+\beta\left(\xi\left(T_{f}\right)\right) \geq \alpha_{s}\left(\widehat{x}_{b}\right)+\beta\left(\widehat{x}_{f}\right) .
$$

Moreover the equality holds if and only if $\xi=\widehat{\xi}$ on $\left[T_{b}, T_{f}\right]$.

\section{The bang arc}

In this section we consider the geometric picture near the bang arc and its lift $\widehat{\lambda}_{\mid\left[0, T_{b}\right]}$ to the cotangent bundle.

If for any $t \in\left[0, T_{b}\right]$ we defi ne the vector fi eld

$$
g_{t} \equiv \widehat{S}_{t *}^{-1} f_{1} \circ \widehat{S}_{t}
$$

then a straightforward calculation gives

$$
\dot{g}_{t}=\widehat{S}_{t *}^{-1}\left[f_{0}, f_{1}\right] \circ \widehat{S}_{t}, \quad \ddot{g}_{t}=\widehat{S}_{t *}^{-1}\left[f_{0}+f_{1},\left[f_{0}, f_{1}\right]\right] \circ \widehat{S}_{t} \quad \forall t \in\left[0, T_{b}\right] .
$$


Remark 4. Since

$$
L_{g_{T_{b}}} L_{g_{T_{b}}} \alpha\left(\widehat{x}_{0}\right)=L_{f_{1}} L_{f_{1}} \alpha_{s}\left(\widehat{x}_{b}\right)>0
$$

$g_{T_{b}}$ is transversal in $\widehat{x}_{0}$ to

$$
M_{0} \equiv \exp \left(-T_{b} f^{+}\right)\left(M_{s}\right)=\left\{z \in M: L_{g_{T_{b}}} \alpha(z)=0\right\} .
$$

Moreover Assumption 2 is equivalent to

$$
L_{\ddot{g}_{T_{b}}} \alpha\left(\widehat{x}_{0}\right)>0 .
$$

As we pointed out in the introduction, $L_{g_{T_{b}}} L_{g_{T_{b}}} \alpha\left(\widehat{x}_{0}\right) \geq 0$ is a necessary condition for the optimality of the bang arc for Problem (1.8) - (1.9). This can be easily seen calculating the second variation of the free-fi xed problem on the bang arc constrained to controls $u$ such that

$$
u(t)= \begin{cases}1 & t \in\left[0, T_{b}-\varepsilon\right] \\ -1 & t \in\left[T_{b}-\varepsilon, T_{b}\right] .\end{cases}
$$

In the following proposition we show that Assumptions 1 and 2, together with (4.1) are suffi cient conditions for $\widehat{\xi}_{\mid\left[0, T_{b}\right]}$ to be a locally optimal trajectory for the free-fi xed problem restricted to the bang arc.

Proposition 2. If Assumptions 7 , 2 and inequality (4.1) are verified, then $\widehat{\xi}_{\mid\left[0, T_{b}\right]}$ is a strict strong local optimizer for Problem (1.8) - (1.9).

Proof: From (4.1) and (4.2), by continuity, there exist $\varepsilon>0$ and a neighborhood $\mathcal{U}$ of $\widehat{x}_{0}$ in $M$ such that the flow $(t, x) \rightarrow \exp t f^{+}(x)$ is defi ned on $\left[0, T_{b}\right] \times \mathcal{U}$, for all $(t, x) \in\left[T_{b}-\varepsilon, T_{b}\right] \times \mathcal{U}$ the following inequalities hold

$$
\begin{aligned}
& L_{g_{t}} L_{g_{t}} \alpha(x) \geq \frac{1}{2} L_{g_{T_{b}}} L_{g_{T_{b}}} \alpha\left(\widehat{x}_{0}\right) \\
& L_{\ddot{g}_{t}} \alpha(x) \geq \frac{1}{2} L_{\ddot{g}_{T_{b}}} \alpha\left(\widehat{x}_{0}\right) \\
& 4 \varepsilon L_{g_{t}} L_{g} \alpha(x)<L_{g_{T_{b}}} L_{g_{T_{b}}} \alpha\left(\widehat{x}_{0}\right),
\end{aligned}
$$

and

$$
L_{g_{t}} \alpha(x)=H_{1}\left(\exp t \vec{H}^{+}(\mathrm{d} \alpha(x))\right)>0 \quad \forall(t, x) \in\left[0, T_{b}-\varepsilon\right) \times \mathcal{U}
$$

We denote

$$
K \equiv \max \left\{\left|L_{g_{t}} L_{g} \alpha(x)\right|:(t, x) \in\left[T_{b}-\varepsilon, T_{b}\right] \times \mathcal{U}\right\} .
$$

We want to prove that $\alpha(\xi(0))-\alpha\left(\widehat{x}_{0}\right) \geq 0$ for any couple $(\xi, u)$ such that $\widehat{\xi}\left(T_{b}\right)=\widehat{x}_{b}$ and $\xi(s) \in$ $\exp s f^{+}(\mathcal{U})$ for any $s \in\left[0, T_{b}\right]$. For such a couple let us defi ne

$$
v_{\varepsilon}(t) \equiv \int_{T_{b}-\varepsilon}^{t}(1-u(s)) \mathrm{d} s, \quad q(t) \equiv \exp \left(-t f^{+}\right)(\xi(t)),
$$

hence

$$
\dot{q}(t)=-(1-u(t)) g_{t}(q(t)), \quad q(0)=\xi(0), \quad q\left(T_{b}\right)=\widehat{x}_{0},
$$


and we can evaluate

$$
\begin{aligned}
& \alpha(\xi(0))-\alpha\left(\widehat{x}_{0}\right)=\int_{T_{b}}^{0} L_{q(s)} \alpha(q(s)) \mathrm{d} s=\int_{0}^{T_{b}}(1-u(s)) L_{g_{s}} \alpha(q(s)) \mathrm{d} s \\
& =\int_{0}^{T_{b}}(1-u(s)) H_{1}\left(\exp s \vec{H}^{+}(\mathrm{d} \alpha(q(s)))\right) \mathrm{d} s \geq \int_{T_{b}-\varepsilon}^{T_{b}}(1-u(s)) L_{g_{s}} \alpha(q(s)) \mathrm{d} s .
\end{aligned}
$$

Integrating by parts and recalling that $q\left(T_{b}\right)=\widehat{x}_{0}$ we get

$$
\begin{aligned}
& \alpha(\xi(0))-\alpha\left(\widehat{x}_{0}\right) \geq-\int_{T_{b}-\varepsilon}^{T_{b}} v_{\varepsilon}(s)\left[L_{g} \alpha(q(s))-\dot{v}_{\varepsilon}(s) L_{g_{s}} L_{g_{s}} \alpha(q(s))\right] \mathrm{d} s \\
& \geq \int_{T_{b}-\varepsilon}^{T_{b}} v_{\varepsilon}(s) \dot{v}_{\varepsilon}(s) \frac{1}{2} L_{g_{T_{b}}} L_{g_{T_{b}}} \alpha\left(\widehat{x}_{0}\right) \mathrm{d} s-\int_{T_{b}-\varepsilon}^{T_{b}} v_{\varepsilon}(s) L_{g} \alpha(q(s)) \mathrm{d} s \\
& \geq \frac{v_{\varepsilon}^{2}\left(T_{b}\right)}{4} L_{g_{T_{b}}} L_{g_{T_{b}}} \alpha\left(\widehat{x}_{0}\right)+\frac{L_{\ddot{g}_{T_{b}}} \alpha\left(\widehat{x}_{0}\right)}{2} \int_{T_{b}-\varepsilon}^{T_{b}}\left(\int_{T_{b}-\varepsilon}^{s} v_{\varepsilon}(t) \mathrm{d} t\right) \mathrm{d} s \\
& \quad-K \int_{T_{b}-\varepsilon}^{T_{b}}\left(\int_{T_{b}-\varepsilon}^{s} v_{\varepsilon}(t) \mathrm{d} t\right) \dot{v}_{\varepsilon}(s) \mathrm{d} s
\end{aligned}
$$

For the last addendum, we have

$$
\begin{aligned}
& K \int_{T_{b}-\varepsilon}^{T_{b}}\left(\int_{T_{b}-\varepsilon}^{s} v_{\varepsilon}(t) \mathrm{d} t\right) \dot{v}_{\varepsilon}(s) \mathrm{d} s=K \int_{T_{b}-\varepsilon}^{T_{b}} v_{\varepsilon}(t)\left(\int_{t}^{T_{b}} \dot{v}_{\varepsilon}(s) \mathrm{d} s\right) \mathrm{d} t \\
\leq & K \int_{T_{b}-\varepsilon}^{T_{b}} v_{\varepsilon}(t) v_{\varepsilon}\left(T_{b}\right) \mathrm{d} t \leq K \varepsilon v_{\varepsilon}^{2}\left(T_{b}\right),
\end{aligned}
$$

so that

$$
\alpha(\xi(0))-\alpha\left(\widehat{x}_{0}\right) \geq \frac{v_{\varepsilon}^{2}\left(T_{b}\right)}{4}\left(L_{g_{T_{b}}} L_{g_{T_{b}}} \alpha\left(\widehat{x}_{0}\right)-4 K \varepsilon\right) \geq 0
$$

because of our choice of $\varepsilon$. To prove that the minimum is strict let us assume $\alpha(\xi(0))=\alpha\left(\widehat{x}_{0}\right)$. If this equality holds, then from (4.8) we get $v_{\varepsilon}\left(T_{b}\right)=0$, i.e. $u(s)=1$ for any $s \in\left[T_{b}-\varepsilon, T_{b}\right]$, so that $\xi(s) \equiv \widehat{\xi}(s)$ for any $s \in\left[T_{b}-\varepsilon, T_{b}\right]$. Since $H_{1}\left(\exp t \vec{H}^{+}(\mathrm{d} \alpha(q(s)))\right)>0$ for any $s \in\left[0, T_{b}-\varepsilon\right]$, equation (4.7) implies $u(s)=1$ also for any $s \in\left[0, T_{b}-\varepsilon\right]$.

Let us now go back to problem (1.1) - (1.2). Without loss of generality we can assume that $\exp \left(-T_{b} f^{+}\right)$is well defi ned on $O\left(\widehat{x}_{b}\right)$, therefore, defi ning $O\left(\widehat{x}_{0}\right)=\exp \left(-T_{b} f^{+}\right)\left(O\left(\widehat{x}_{b}\right)\right)$ and $O_{s}\left(\widehat{x}_{0}\right)=\exp \left(-T_{b} f^{+}\right)\left(O_{s}\left(\widehat{x}_{b}\right)\right)$ we have that for any $x \in O\left(\widehat{x}_{0}\right)$ there exists one and only one couple $(a, z) \in(-\bar{b}, \bar{b}) \times O_{s}\left(\widehat{x}_{0}\right)$ such that

$$
x=\exp a g_{T_{b}}(z) .
$$

Remark 5. Possibly restricting $O_{s}\left(\widehat{x}_{0}\right)$, Assumption 3 is equivalent to

$$
L_{\dot{g}_{b}} \alpha(z) \leq 0 \quad \forall z \in O_{s}\left(\widehat{x}_{0}\right)
$$

The map

$$
\widetilde{\alpha}: O\left(\widehat{x}_{0}\right) \rightarrow \widetilde{\alpha}_{s} \circ \exp T_{b} f^{+} .
$$

is constant along the integral lines of $g_{T_{b}}$, so that

$$
L_{g_{T_{b}}} \widetilde{\alpha}(x)=0 \text { for any } x \in O\left(\widehat{x}_{0}\right) \text {. }
$$


Moreover it is not diffi cult to see that $L_{\mathbf{v}} \alpha(z)=L_{\mathbf{v}} \widetilde{\alpha}(z)$ for any $z \in O_{s}\left(\widehat{x}_{0}\right)$ and for any $\mathbf{v} \in T_{z} M$. Without loss of generality we can assume that $O\left(\widehat{x}_{0}\right)$ is contained in the neighborhood $\mathcal{U}$, defi ned in the proof of Proposition of; therefore from now on we assume that equations (4.3) - (4.4) - (4.5) and (4.6) hold. Moreover, possibly restricting $\varepsilon>0$ and $O\left(\widehat{x}_{0}\right)$ we assume

1. $\alpha(x) \geq \widetilde{\alpha}(x)$ for any $x$ in $O\left(\widehat{x}_{0}\right)$;

2. $L_{g_{T_{b}}} L_{g_{T_{b}}} \alpha(z)-\varepsilon L_{\exp \left(-a g_{T_{b}}\right)_{*} g_{t} \circ \exp a g_{T_{b}}} L_{g_{T_{b}}} \alpha(z)>0 \forall(t, x) \in\left[T_{b}-\varepsilon, T_{b}\right) \times O\left(\widehat{x}_{0}\right)$;

3. $L_{\ddot{g}_{t}} \widetilde{\alpha}(x)>0 \quad \forall(t, x) \in\left[T_{b}-\varepsilon, T_{b}\right) \times O\left(\widehat{x}_{0}\right) ;$

4. $L_{\left[g_{T_{b}}, g_{b}\right]} \widetilde{\alpha}(x)>0 \quad \forall x \in O\left(\widehat{x}_{0}\right)$.

With such a choice of $\varepsilon$ and $O\left(\widehat{x}_{0}\right)$, let us defi ne

$$
\begin{aligned}
& M:(t, a, z) \in\left[0, T_{b}\right] \times(-\bar{b}, \bar{b}) \times O_{s}\left(\widehat{x}_{0}\right) \rightarrow H_{1}\left(\exp t \vec{H}^{+}\left(\mathrm{d} \alpha\left(\exp a g_{T_{b}}(z)\right)\right)\right) \in \mathbb{R}, \\
& \widetilde{M}:(t, a, z) \in\left[0, T_{b}\right] \times(-\bar{b}, \bar{b}) \times O_{s}\left(\widehat{x}_{0}\right) \rightarrow H_{1}\left(\exp t \vec{H}^{+}\left(\mathrm{d} \widetilde{\alpha}\left(\exp a g_{T_{b}}(z)\right)\right)\right) \in \mathbb{R} .
\end{aligned}
$$

Assumption 3 is crucial in the proofs of the following two "twin" Lemmata.

Lemma 2. If Assumption 3 holds, then

$$
M(t, a, z) \geq 0 \quad \text { for any }(t, a, z) \in\left(T_{b}-\varepsilon, T_{b}\right) \times[0, \bar{b}] \times O_{s}\left(\widehat{x}_{0}\right) .
$$

Proof: Let us write the Taylor expansion of $M$ in a neighborhood of $(t, a, z)=\left(T_{b}, 0, \widehat{x}_{0}\right)$.

$$
\begin{aligned}
M(t, a, z) & =L_{g_{t}} \alpha\left(\exp a g_{T_{b}}(z)\right)=L_{g_{T_{b}}} \alpha\left(\exp a g_{T_{b}}(z)\right) \\
& +\left(t-T_{b}\right) L_{\dot{g}_{b}} \alpha\left(\exp a g_{T_{b}}(z)\right)+\frac{\left(t-T_{b}\right)^{2}}{2} L_{\ddot{g}_{\theta}} \alpha\left(\exp a g_{T_{b}}(z)\right) \quad\left(\text { for some } \theta \in\left(t, T_{b}\right)\right) \\
& =L_{g_{T_{b}}} \alpha(z)+a L_{g_{T_{b}}} L_{g_{T_{b}}} \alpha\left(\exp \beta_{1} g_{T_{b}}(z)\right)+\left(t-T_{b}\right) L_{g_{b}} \alpha\left(\exp a g_{T_{b}}(z)\right) \\
& +\frac{\left(t-T_{b}\right)^{2}}{2} L_{\ddot{g}_{\theta}} \alpha\left(\exp a g_{T_{b}}(z)\right) \quad\left(\text { for some } \beta_{1} \text { intermediate between } 0 \text { and } a\right) \\
& =a\left[L_{g_{T_{b}}} L_{g_{T_{b}}} \alpha\left(\exp \beta_{1} g_{T_{b}}(z)\right)+\left(t-T_{b}\right) L_{g_{T_{b}}} L_{\dot{g}_{b}} \alpha\left(\exp \beta_{2} g_{T_{b}}(z)\right)\right] \\
& +\left(t-T_{b}\right)\left[L_{\dot{g}_{b}} \alpha(z)+\frac{t-T_{b}}{2} L_{\ddot{g}_{\theta}} \alpha\left(\exp a g_{T_{b}}(z)\right)\right]
\end{aligned}
$$

( for some $\beta_{2}$ intermediate between 0 and $a$ ).

Hence Assumption 3 and equation (4.5) give the result.

Lemma 3. If Assumption 3 holds, then

$$
\widetilde{M}(t, a, z) \geq 0 \quad \text { for any }(t, a, z) \in\left(T_{b}-\varepsilon, T_{b}\right) \times[-\bar{b}, 0] \times O_{s}\left(\widehat{x}_{0}\right) .
$$

Proof: Repeating the proof of Lemma 2 we get

$$
\widetilde{M}(t, a, z)=\left(t-T_{b}\right)\left[2 a L_{\left[g_{T_{b}}, \dot{g}_{b}\right.} \widetilde{\alpha}\left(\exp \beta_{2} g_{T_{b}}(z)\right)+\frac{t-T_{b}}{2} L_{\ddot{g}_{\theta}} \widetilde{\alpha}\left(\exp a g_{T_{b}}(z)\right)+2 L_{\dot{g}_{b}} \widetilde{\alpha}(z)\right]
$$

for some $\theta \in\left(T_{b}-\varepsilon, T_{b}\right)$ and some $\beta_{2}$ intermediate between 0 and $a$. Due to the restriction on $\varepsilon>0$ and $O\left(\widehat{x}_{0}\right)$, we get the result. 
The following Lemma describes the behaviour of the trajectories of control system (1.2) and its proof does not require Assumption 3 .

Lemma 4. Let $(\xi, u)$ be an admissible couple such that $\xi(t) \in \exp t f^{+}\left(O\left(\widehat{x}_{0}\right)\right)$ for any $t \in\left[0, T_{b}\right]$. Define $q(t) \equiv \exp \left(-t f^{+}\right)(\xi(t)) \in O\left(\widehat{x}_{0}\right)$ and let $(a(t), z(t)) \in(-\bar{b}, \bar{b}) \times O_{s}\left(\widehat{x}_{0}\right)$ be defined by $q(t)=$ $\exp a(t) g_{T_{b}}(z(t))$. Then $a: t \in\left[T_{b}-\varepsilon, T_{b}\right] \rightarrow a(t) \in \mathbb{R}$ is a monotone non-increasing function.

Proof: Differentiating the identity $z(t)=\exp \left(-a(t) g_{T_{b}}\right)(q(t))$ we get

$$
\dot{z}(t)=-\dot{a}(t) g_{T_{b}}(z(t))-(1-u(t)) \exp \left(-a(t) g_{T_{b}}\right)_{*} g_{t} \circ \exp a(t) g_{T_{b}}(z(t))
$$

Also differentiating the identity $L_{g_{T_{b}}} \alpha(z(t))=0$, we get $L_{z_{z}(t)} L_{g_{T_{b}}} \alpha(z(t))=0$. Hence we have

$$
-\dot{a}(t) L_{g_{T_{b}}} L_{g_{T_{b}}} \alpha(z(t))-(1-u(t)) L_{\exp \left(-a(t) g_{T_{b}}\right) * g_{t} \circ \exp a(t) g_{T_{b}}} L_{g_{T_{b}}} \alpha(z(t))=0
$$

which gives

$$
\dot{a}(t)=-(1-u(t))\left\{1+\left(t-T_{b}\right) \frac{L_{\exp \left(-a(t) g_{T_{b}}\right)_{*} g_{\theta(t)} \exp a(t) g_{T_{b}}} L_{g_{T_{b}}} \alpha(z(t))}{L_{g_{T_{b}} L_{g_{b}}} \alpha(z(t))}\right\}
$$

which is non-positive due to the restrictions on $\varepsilon$ and $O\left(\widehat{x}_{0}\right)$.

\section{Proof of Theorem 1}

Let $(\xi, u)$ be an admissible couple such that $\xi(t) \in \exp t f^{+}\left(O\left(\widehat{x}_{0}\right)\right)$ for any $t \in\left[0, T_{b}\right]$ and the graph of $\xi_{\mid\left[T_{b}, T_{f}\right]}$ belongs to the neighborhood $\mathcal{V}$ defi ned in Lemma[1. We want to show

$$
\alpha(\xi(0))-\alpha\left(\widehat{x}_{0}\right) \geq \widetilde{\alpha}_{s}\left(\xi\left(T_{b}\right)\right)-\alpha_{s}\left(\widehat{x}_{b}\right),
$$

or, equivalently with the notation of Lemma 4

$$
\alpha(q(0)) \geq \widetilde{\alpha}\left(q\left(T_{b}\right)\right) .
$$

Since

$$
\alpha(q(0))-\alpha\left(q\left(T_{b}\right)\right)=\int_{T_{b}}^{0} \frac{\mathrm{d}}{\mathrm{d} s} \alpha(q(s)) \mathrm{d} s=\int_{0}^{T_{b}}(1-u(s)) M(s, a(s), z(s)) \mathrm{d} s,
$$

then, if $M(s, a(s), z(s)) \geq 0, \forall s \in\left[0, T_{b}\right]$, we get

$$
\alpha(q(0)) \geq \alpha\left(q\left(T_{b}\right)\right) \geq \widetilde{\alpha}\left(q\left(T_{b}\right)\right) .
$$

If there exists $\bar{t} \in\left(T_{b}-\varepsilon, T_{b}\right)$ such that $M(\bar{t}, a(\bar{t}), z(\bar{t}))=0$ then, from Lemma $2, a(\bar{t})<0$,

$$
\alpha(q(0)) \geq \alpha(q(\bar{t}))+\int_{0}^{\bar{t}}(1-u(s)) M(s, a(s), z(s)) \mathrm{d} s \geq \alpha(q(\bar{t})) \geq \widetilde{\alpha}(q(\bar{t}))
$$

so that

$$
\widetilde{\alpha}(q(\bar{t}))-\widetilde{\alpha}\left(q\left(T_{b}\right)\right)=\int_{\bar{t}}^{T_{b}}(1-u(s)) \widetilde{M}(s, a(s), z(s)) \mathrm{d} s .
$$

Since $a(s)$ is decreasing by Lemma 4 and $a(\bar{t}) \leq 0$, applying Lemma 3 equation (5.1) is proved. 
Considering also the result of Lemma 1] we obtain

$$
\begin{aligned}
& \alpha(\xi(0))+\beta\left(\xi\left(T_{f}\right)\right)-\left(\alpha\left(\widehat{x}_{0}\right)+\beta\left(\widehat{x}_{f}\right)\right)= \\
& =\left[\alpha(\xi(0))-\widetilde{\alpha}_{s}\left(\xi\left(T_{b}\right)\right)\right]+\left[\widetilde{\alpha}_{s}\left(\xi\left(T_{b}\right)\right)+\beta\left(\xi\left(T_{f}\right)\right)-\alpha_{s}\left(\widehat{x}_{b}\right)-\beta\left(\widehat{x}_{f}\right)\right] \geq 0 .
\end{aligned}
$$

To complete the proof we have to show that the minimum is strict. Assume equality holds in (5.3), by equation (5.1) and Lemma 1 we obtain

$$
\begin{aligned}
& \widetilde{\alpha}_{s}\left(\xi\left(T_{b}\right)\right)+\beta\left(\xi\left(T_{f}\right)\right)-\alpha_{s}\left(\widehat{x}_{b}\right)-\beta\left(\widehat{x}_{f}\right)=0 \\
& \alpha(\xi(0))-\widetilde{\alpha}_{s}\left(\xi\left(T_{b}\right)\right)=0
\end{aligned}
$$

From equation (5.4) and Lemma 1]we obtain

$$
\xi=\widehat{\xi} \text { on }\left[T_{b}, T_{f}\right]
$$

hence $\xi\left(T_{b}\right)=\widehat{x}_{b}$. Finally, from equation (5.5) and Proposition 2 we obtain

$$
\xi=\widehat{\xi} \text { on }\left[0, T_{b}\right] \text {. }
$$

\section{Final remarks}

The result of Theorem 1 is not completely satisfactory because of Assumption B. Indeed it is not clear if it is "almost necessary" like the other ones. Assumption 3 allows us to prove that "near" the singular point $\widehat{\lambda}\left(T_{b}\right)$, the flow associated to the Hamiltonian $H^{+}$starting from the union of two suitable "half Lagrangean sub-manifolds" remains in the region where $H^{+}=H^{\max }$ for all $t \in\left[0, T_{b}\right]$, see Lemmata 2, 3. Therefore the flow of the vector fi eld $f^{+}$starting from a neighborhood of $\widehat{\xi}(0)$ is a flow of non intersecting extremals covering a neighborhood of the bang arc.

So far we have not been able to fi nd a flow with such properies without Assumption 3 . More investigation is in progress in order to understand the role of Assumption 3 . However, by means of this result it is possible to prove suffi cient conditions for a bang-singular arc in the minimum time problem and in the Bolza problem with fi xed end-points requiring the extended second variation on the singular arc to be coercive on a larger space.

\section{References}

[1] Andrei A. Agrachev, Gianna Stefani, and PierLuigi Zezza. An invariant second variation in optimal control. Internat. J. Control, 71(5):689-715, 1998.

[2] Andrei A. Agrachev, Gianna Stefani, and PierLuigi Zezza. Strong optimality for a bang-bang trajectory. SIAM J. Control Optimization, 41:991-1014, 2002.

[3] Magnus R. Hestenes. Application of the theory of quadratic forms in Hilbert spaces to calculus of variations. Pacific J. Math. 1:521-581, 1951.

[4] Brian P. Molinari. Non-negativity of a quadratic functional. SIAM J. Control , 13:262-271, 1975.

[5] Gianna Stefani and PierLuigi Zezza. Constrained Regular LQ-Control Problems. SIAM J. Control Optimization, 35:876-900, 1997.

[6] Gianna Stefani. Optimality conditions for a singular extremal: the single-input case. Preprint, 2004.

[7] Vera Zeidan. The Riccati equation for optimal control problems with mixed state-control constraints: Necessity and sufficiency. SIAM J. Control Optimization, 32:1297-1321, 1994. 